\title{
Detection and Classification of Stator Short-Circuit Faults in Three-Phase Induction Motor
}

\section{*ABDULLATEEF, AI; FAGBOLAGUN, OS; SANUSI, MF; AKOREDE, MF; AFOLAYAN, MA}

Department of Electrical and Electronics Engineering, University of Ilorin, Ilorin, Kwara State, Nigeria

${ }^{*}$ Corresponding Author Email: abd_lateef.aii@unilorin.edu.ng

\begin{abstract}
Induction motors are the backbone of the industries because they are easy to operate, rugged, economical and reliable. However, they are subjected to stator's faults which damage the windings and consequently lead to machine failure and loss of revenue. Early detection and classification of these faults are important for the effective operation of induction motors. Stators faults detection and classification based on wavelet Transform was carried out in this study. The feature extraction of the acquired data was achieved using lifting decomposition and reconstruction scheme while Euclidean distance of the Wavelet energy was used to classify the faults. The Wavelet energies increased for all three conditions monitored, normal condition, inter-turn fault and phase-to-phase fault, as the frequency band of the signal decreases from D1 to A3. The deviations in the Euclidean Distance of the current of the Wavelet energy obtained for the phase-to-phase faults are $99.1909,99.8239$ and 87.9750 for phases A and B, $\mathrm{A}$ and C, B and C respectively. While that of the inter-turn faults in phases A, B and C are 77.5572, 61.6389 and 62.5581 respectively. Based on the Euclidean distances of the faults, $\mathrm{D}_{\mathrm{f}}$ and normal current signals, three classification points were set: $\mathrm{K}_{1}=0.60 \times 10^{2}, \mathrm{~K}_{2}=0.80 \times 10^{2}$ and $\mathrm{K}_{3}=1.00 \times 102$. For $\mathrm{K}_{2} \geq \mathrm{D}_{\mathrm{f}} \geq \mathrm{K}_{1}$ inter-turn faults is identified and for $K_{3} \geq D_{f} \geq K_{2}$ phase to phase fault identified. This will improve the induction motors stator's fault diagnosis.
\end{abstract}

DOI: $\underline{\text { https://dx.doi.org/10.4314/jasem.v24i3.3 }}$

Copyright: Copyright (C) 2020 Abdullateef et al. This is an open access article distributed under the Creative Commons Attribution License (CCL), which permits unrestricted use, distribution, and reproduction in any medium, provided the original work is properly cited.

Dates: Received: 25 January 2020; Revised: 11 February 2020; Accepted: 22 February 2020

Keywords: induction motor, stator fault classification, data acquisition system, Discrete Wavelet Transform

Induction motors are widely used as industrial drives because they are rugged, reliable and economical and easy to operate (Dubravko, 2015). Despite the aforementioned advantages, they are susceptible to various types of faults which hinders their performances. The most common faults in induction motors are bearing faults, stator faults, rotor faults and a combination of other faults. These faults are statistically categorised as $40 \%, 37 \% 10 \%$ and $13 \%$ respectively (Bonaldi et al., 2012; Verrucchi, 2008). Increasing demand for greater performance, protection and consistency of industrial systems have awakened the need for induction motor fault analysis and health monitoring (Siddiqui et al., 2014) . Earlier detection and classification of induction motor faults are essential for the efficient operation of the machines and reduction of revenue loss incurred due to faulty condition. The cost incurred due to unexpected breakdowns is very high as a lot of machines depend on the continuous and parallel operation of induction motors in the industries (Subha, 2018). Moreover, the safety of the operator is guaranteed when faults are detected early and isolated. Consequently, the monitoring of faults in induction motors are becoming more and more important issues in the field of electrical machines as new data processing techniques and new technologies for electrical drives emerge (Nagarajan and Reddy, 2013). Current and vibration data are one of the most important parameters used for condition monitoring and fault analysis since they possess the dynamic information of the motor (Bhowmik et al., 2013). Although, their performance depends on the efficiency of the technique used in the processing of the data. Several techniques have been proposed and can be categorised as model based techniques (Isermann, 2015; Sahraoui et al., 2010), soft computing technique (Bendjama et al., 2013; Liu et al., 2018) and signal processing technique (Glowacz et al., 2018; Palácios et al., 2015).

Over the years, signal processing techniques such as Fourier Transform (FT), Fast Fourier Transform (FFT), Short-Time Fourier Transform (STFT) and Wavelet Transform (WT) have been used for signals analysis. These techniques except the latter have their set-backs in non-stationary signal analysis (Bhowmik et al., 2013). WT is able to diagnose faults in the transient conditions with improved frequency 
resolution. Besides, it gives the time and frequency information of the signal simultaneously (Polikar, 2008). WT uses its decomposition ability to obtain the approximation and detail coefficient of the original signal with different frequency bands by using sequential low-pass and high-pass filters. Thus it has applied to detect faults in induction machines (Granda et al., 2018). Bendjama et al. (2013) proposed Wavelets and principal component analysis for vibration monitoring of rotating machinery. The vibration data which was decomposed using Wavelet decomposition method were used as the input to the Principal Component Analysis for fault identification.

Ray et al. (2016) proposed the detection of fault disturbances using Wavelet Transform and Independent Component Analysis. Voltage signal extracted at the power control centre was processed through this technique under different fault and operating scenarios. Signal monitoring technique for broken bars fault diagnosis based on uncertainty bounds violation for three-phase induction motors has been proposed (Mustafa et al., 2012). The fault detection and diagnosis were performed in a two-steps procedure where the parameters of the healthy motor are identified on the basis of the set membership identification scheme and online fault diagnosis was performed on the basis of bounds violation conditions. Liang et al. (2018) proposed detection of inter-turn short-circuits in permanent magnet synchronous machine (PMSM) using stator current and vibration signals. A time-frequency analysis based on improved
Wavelet packet transform was used for fault detected. Furthermore, fault feature extraction of the mechanical irregularity of induction motors bearing proposed by He et al. (2015) was based on Ensemble Super-wavelet transform (ESW). The ESW is based on the combination of Tuneable Q-factor Wavelet Transform (TQWT) and Hilbert transform such that fault feature adaptability is enabled. Cusidó et al. (2011) proposed the problems of fault detection of induction motors under a variable load torque. The Discrete Wavelet Transform (DWT) was used and compared to the motor current signature analysis. Motor Current Signature Analysis and Continuous Wavelet Transform (CWT) approach to diagnosis the broken bars in a three-phase induction has been proposed (Granda et al., 2017). Thus, the objective of this study is to detect and classify the stators faults in a three phase iduction motor using current data acquired and analysed based on Wavelt Transform.

\section{MATERIALS AND METHODS}

Acquisition of normal and faults current data: Data acquisition system was developed to acquire the current data used for this study. Detailed development which includes the rewinding of three-phase induction motor; circuit design and components layout; selection of appropriate components; programming and compilation of the programmed code on the Arduino board; Calibration of sensors and testing of the DAQ system can be found in (Abdullateef et al., 2019). Figure 1 shows the experimental set-up.

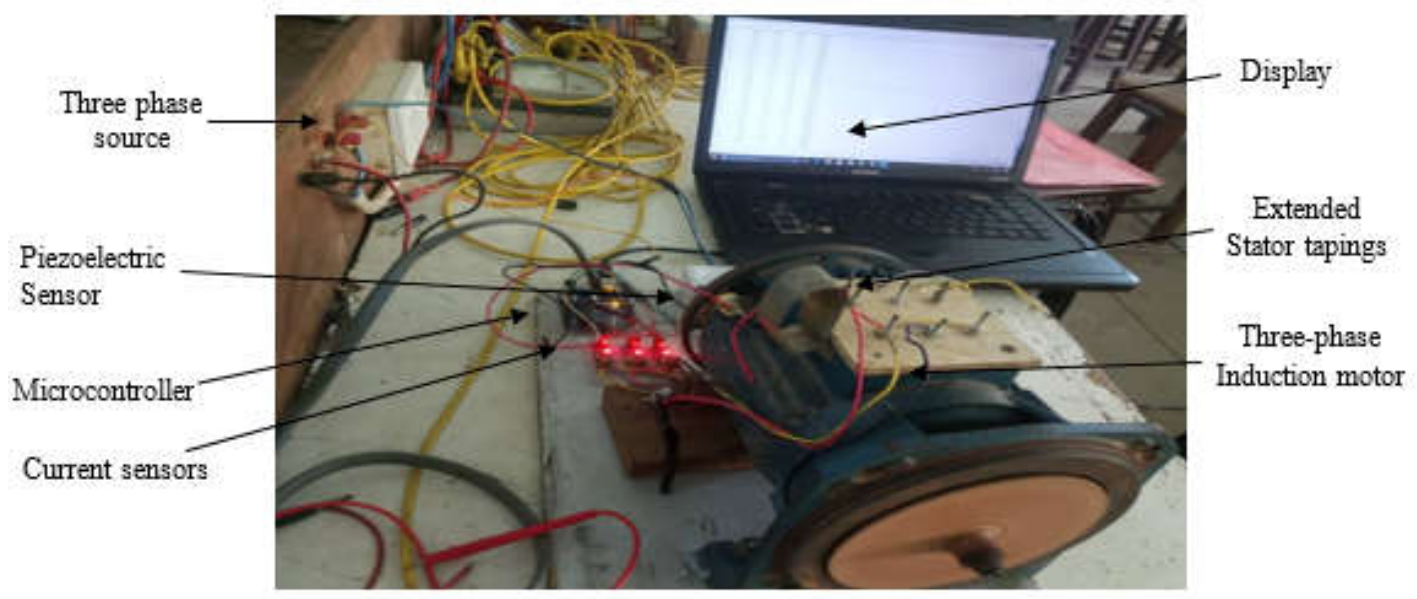

Fig 1. Experimental Test Rig for Data Acquisition

Discrete Wavelet Transform: The Discrete Wavelet Transform (DWT) method is often referred to as dialect scaling and shifting. It eliminates redundancy in coefficient and still retains the output of the transform to yield the same number of coefficient as the length of the input signal, therefore, requires less memory (Tangborn, 2013).

Let $x(t)$ be the signal, then the DWT is defined as 


$$
D W T(x, a, b)=\frac{1}{\sqrt{a_{o}^{j}}} \sum_{k} x(t) \psi\left(\frac{t-k b_{o}^{j}}{a_{o}^{j}}\right)
$$

Where; $\psi(k)$ is the mother wavelet which contains the scaling (dilation) and translation (time shift) constants $a, b$ respectively? $k$ and $j$ are integer variables.

The base signal scaling in DWT is set to 2 and different scales can be obtained by raising this base scale to integer values represented as $2^{j}$ where $(j=1$, $2,3 \ldots \mathrm{n})$. The translation occurs at integer multiple represented as $2_{m}^{j}$ where $(m=1,2,3 \ldots n)$.

Selection of mother wavelet and Decomposition level: Daubechies mother wavelet was used in this study based on its ability to extract all signal energies than other mother wavelet and has a function that supports compatibility (Kessler et al., 2008). Equation 2 is the condition for selecting the minimum number of decomposition levels that is necessary for obtaining an approximation signal so that the upper limit of its associated frequency band is under the fundamental frequency (Mallat, 1989). The frequency band of the signal must be at least twice the sampling frequency (Nyquist theory).

$$
2^{-\left(n_{L s}+1\right)} f_{s}<f
$$

Where; $n_{L s}$ is the number of decomposition levels, $f_{s}$ is the sampling frequency and $f$ is the fundamental frequency.

The original signal is decomposed into two scales. The large scales allow us to know the signal characteristic at low frequencies known as approximation wavelet coefficients $C a_{j}$, whereas the small scales show the signal characteristics at high frequencies known as the detail wavelet coefficients $C d_{j}$. The wavelet coefficients are transformed into the approximation and detail coefficients to be represented in the timefrequency spectrum. These coefficients are displayed to perform a comparison between the motor currents data and vibration data at normal and fault conditions. The approximation and the detail wavelet coefficients can be expressed as equation 3 and 4 respectively.

$$
C a_{j}[n]=\sum_{l_{n}} f_{l_{0}}\left[2 n-l_{n}\right] \cdot x[n]
$$

$$
C d_{j}[n]=\sum_{l_{n}} f_{l_{1}}\left[2 n-l_{n}\right] \cdot x[n]
$$

Where; $x[n]$ is the sampled signal, $f_{l_{0}}$ is the low pass filter and $f_{l_{1}}$, is the high pass filter. $l_{n}$ is the wavelength, $C a_{j}$ and $C d_{j}$ are the approximation and detail coefficients respectively.

The fault classification algorithm is proposed based on the wavelet energy at different levels. which can be decomposed using Parseval's theorem (Omerhodzic et al., 2013).

$$
\begin{gathered}
E_{D i}=\sum_{j=1}^{N}\left|D_{i j}\right|^{2}, i=1, \ldots, l \\
E_{A i}=\sum_{j=1}^{N}\left|A_{i j}\right|^{2}
\end{gathered}
$$

Where $i=1, \ldots, l$ is the wavelet decomposition level from level 1 to level $l$. $N$ is the number of the coefficients of detail and approximate at each decomposition level. $E_{D i}$ is the energy of the detail and $E_{A i}$ is the energy of the approximate at decomposition level $l$.

In order to address energy imbalance, the energies of the wavelet coefficients are normalized using equation 7.

$$
N_{E_{i}}(k)=\frac{E_{i}(k)-\mu\left(E_{i}\right)}{\sigma\left(E_{i}\right)}
$$

Where $\mu$ is the mean value of the original signal and $\sigma$ is the corresponding standard deviation of the signal, $E_{i}(k)$ is the Wavelet Energy of all decomposition level at a fault type.

The Euclidean Distance among every fault state and the normal state is used as a criterion for fault classification is expressed in equation 8 .

$$
E D_{r}=\sqrt{\sum_{r}\left[N_{E_{f}}(r)-N_{E_{h}}(r)\right]^{2}}
$$

Where; $N_{E_{f}}$ and $N_{E_{h}}$ are the Normalised Wavelet energies at faults and normal conditions.

Lifting scheme technique for data feature extraction: Lifting scheme is a suitable tool used to perform a filtering process due to the unwanted components noticed in the raw data signal. It accomplishes the 
design and implementation of the bi-orthogonal wavelet transforms with downsampling and interpolation factor $N=2$.

Generally, the lifting mode consists of two parts; the decomposition and reconstruction parts. The decomposition is to obtain the approximation and detail coefficients of the original signal. It includes splitting, prediction and updating. The reconstruction part is the reverse of the decomposition. The lifting scheme flowchart is as shown in Figure 2.

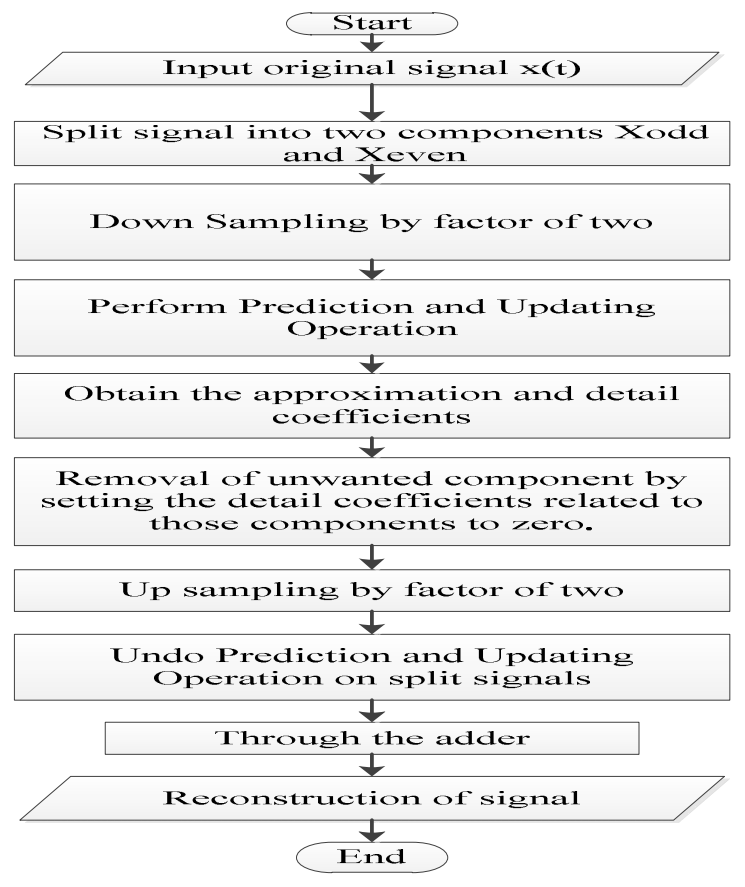

Fig 2: Lifting scheme flowchart

\section{RESULTS AND DISCUSSION}

Three conditions of the motor were monitored: motor under normal condition, motor under phase-to-phase fault condition and inter-turn fault condition. Typical samples of the acquired data under these conditions are shown in (Abdullateef et al., 2019). The multiresolution analysis was implemented using the Daubechies mother wavelet of the order $4(\mathrm{db} 4)$ and order $8(\mathrm{db} 8)$. The same mother wavelet with the same decomposition level is applied to each signal. The result of $\mathrm{db} 4$ and $\mathrm{db} 8$ decomposition of current in phase A is shown in Figure 3. The wavelet decomposition of current shown in Figure 4 depict an instance of fault. After decomposition with $\mathrm{db} 4$ and $\mathrm{db} 8$, the wavelet energy for approximation and detail coefficients were obtained using equation 6 and 7 respectively. The result for normal condition and short-circuit fault are illustrated in Table 1-Table 3. It is observed from Table 1 - Table 3 that as the frequency band of the signal decreases from D1 to A3, the wavelet energies increases for all the three condition monitored (normal, inter-turn fault and phase-to-phase fault) when db8 mother wavelet is used.
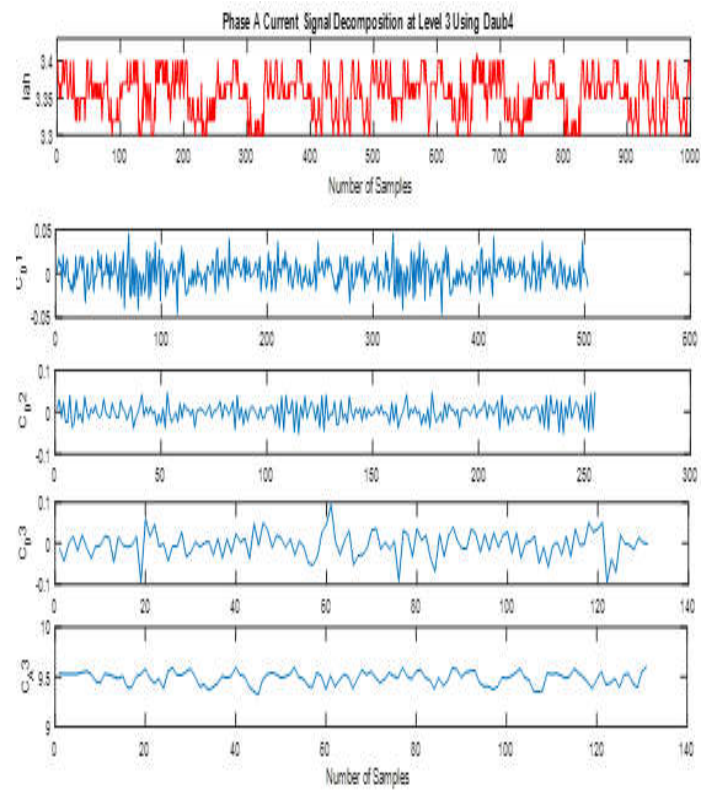

Fig 3. Phase A normal state current signal details and approximation coefficients

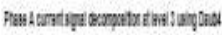
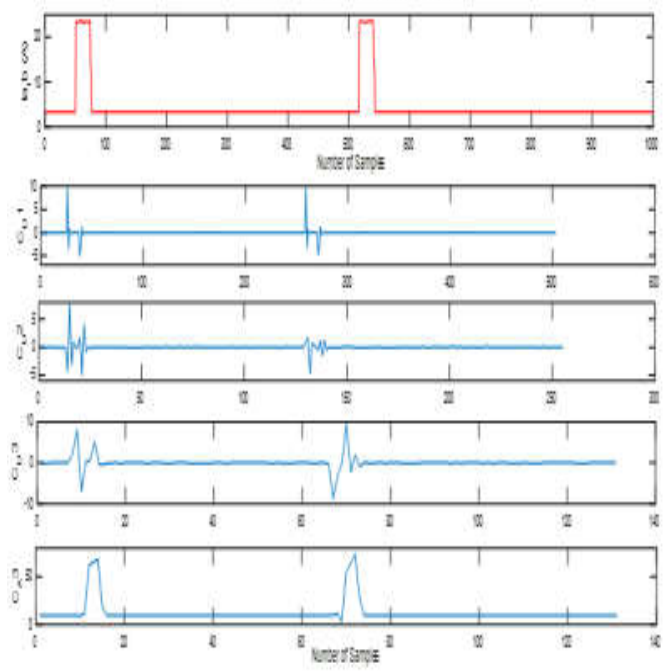

Fig 4. Phase A current signal with fault details and approximation coefficients

The phase-to-phase fault always has more energy than the inter-turn fault. In addition, among the three detail energy coefficients, D3 $(20.75 \mathrm{~Hz}-41.5 \mathrm{~Hz})$ has the highest energy. Figure 5 and Figure 6 explains the increase in approximate coefficient wavelet energy of $\mathrm{db} 8$ over $\mathrm{db} 4$. 
Table 1: wavelet energy for currents computed for two different levels of Daubechies families (i.e. db4 and db8) at three decomposition level under normal motor condition

\begin{tabular}{ccccccccc}
\hline & \multicolumn{3}{c}{$\mathrm{Db} 4$} & \multicolumn{5}{c}{$\mathrm{Db} 8$} \\
${$\cline { 2 - 4 }$} }$ & $E D_{2}$ & $E D_{3}$ & $E A_{3}$ & $E D_{1}$ & $E D_{2}$ & $E D_{3}$ & $E A_{3}$ \\
$I_{a_{-} h}$ & 0.1107 & 0.1219 & 0.1298 & $1.1801 \mathrm{e}+04$ & 0.1006 & 0.1026 & 0.1716 & $1.2441 \mathrm{e}+04$ \\
$I_{b_{-} h}$ & 0.1169 & 0.1628 & 0.2011 & $1.0181 \mathrm{e}+04$ & 0.1154 & 0.1168 & 0.3223 & $1.0719 \mathrm{e}+04$ \\
$I_{c_{-} h}$ & 0.1125 & 0.0896 & 0.1148 & $0.7479 \mathrm{e}+04$ & 0.0832 & 0.1034 & 0.1439 & $0.7886 \mathrm{e}+04$ \\
\hline
\end{tabular}

Table 2: wavelet energy for currents computed for two different levels of Daubechies families (i.e. db4 and db8) at three decomposition

\begin{tabular}{ccccccccc}
\multicolumn{1}{c}{ level under inter-turn fault conditions. } \\
\cline { 2 - 9 } & $E D_{1}$ & $E D_{2}$ & $E D_{3}$ & $E A_{3}$ & $E D_{1}$ & $E D_{2}$ & $E D_{3}$ & $E A_{3}$ \\
\hline$I_{a_{-} i a}$ & 51.9310 & 21.7129 & 76.1864 & $1.8354 \mathrm{e}+04$ & 25.1167 & 50.8202 & 81.9115 & $1.8982 \mathrm{e}+04$ \\
$I_{b_{-} i b}$ & 59.7307 & 31.6654 & 82.5818 & $1.6017 \mathrm{e}+04$ & 27.5062 & 71.8678 & 99.7031 & $1.6537 \mathrm{e}+04$ \\
$I_{c_{-} i c}$ & 33.3889 & 22.3142 & 39.6057 & $1.1865 \mathrm{e}+04$ & 18.5309 & 40.6846 & 59.6557 & $1.4255 \mathrm{e}+04$ \\
\hline
\end{tabular}

Table 3: wavelet energy for currents computed for two different levels of Daubechies families (i.e. db4 and db8) at three decomposition

\begin{tabular}{ccccccccc}
\multicolumn{7}{c}{ level under phase to phase faults. } \\
\hline & \multicolumn{7}{c}{$\mathrm{Db} 4$} & \multicolumn{5}{c}{$\mathrm{Db8}$} \\
\cline { 2 - 9 }$I_{a_{-} a b}$ & 56.2067 & 36.0026 & 68.5237 & $1.7442 \mathrm{e}+04$ & 24.9539 & 62.0664 & 86.6804 & $1.8065 \mathrm{e}+04$ \\
$I_{b_{-} a b}$ & 64.0175 & 34.9103 & 59.4115 & $1.6691 \mathrm{e}+04$ & 28.0431 & 65.7756 & 89.3175 & $1.7211 \mathrm{e}+04$ \\
$I_{a_{-} a c}$ & 64.9755 & 40.8524 & 76.7285 & $1.8586 \mathrm{e}+04$ & 29.8695 & 66.5296 & 101.1201 & $1.9201 \mathrm{e}+04$ \\
$I_{c_{-} a c}$ & 32.6117 & 17.5738 & 38.2159 & $1.1309 \mathrm{e}+04$ & 14.9295 & 33.6007 & 50.8498 & $1.4698 \mathrm{e}+04$ \\
$I_{b_{-} b c}$ & 61.0422 & 36.7533 & 68.2454 & $1.6862 \mathrm{e}+04$ & 28.1979 & 63.0591 & 93.1956 & $1.7381 \mathrm{e}+04$ \\
$I_{c \_b c}$ & 67.0498 & 45.9540 & 71.8705 & $1.3785 \mathrm{e}+04$ & 30.1379 & 70.7723 & 85.2841 & $1.4191 \mathrm{e}+04$ \\
\hline
\end{tabular}

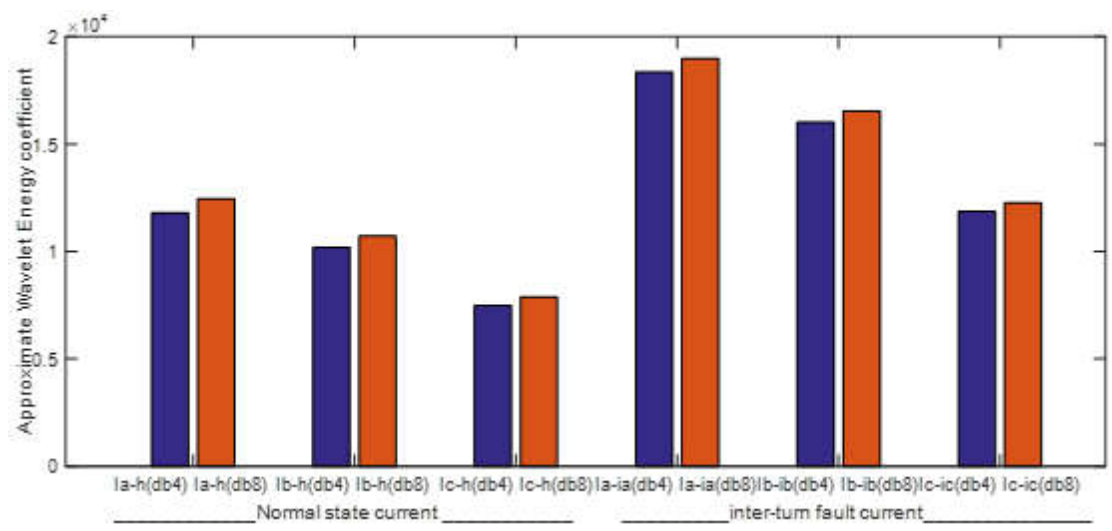

Fig 5. Approximation wavelet energy for healthy and inter-turn current obtained for db4 and db8

The higher the wavelet energy value the more significant the characteristics of the energy. The normalised current and vibration Wavelet Energy for all types of faults and normal state are represented in Table 4 to Table 6 using equation 7. This energy gives the same energy value at all three levels of decomposition, therefore, single energy value is selected to represent the wavelet energy of each fault signal. Figure 7 is the Euclidean Distance obtained using equation 8 showing substantial classification 
between the faults. Phase-to-phase short circuit faults provide more distance variation from the healthy state current compared to inter-turn faults. Phase-to-phase fault current in phase $\mathrm{A}$ and $\mathrm{C}$ contributes more Euclidean Distance of $0.99824 \times 10^{2}$ from the normal motor current, inter-turn fault current in phase B contributes lesser distance of $0.61639 \times 10^{2}$ from the healthy current. This explains the severity of a phase-to-phase fault over inter-turn fault.

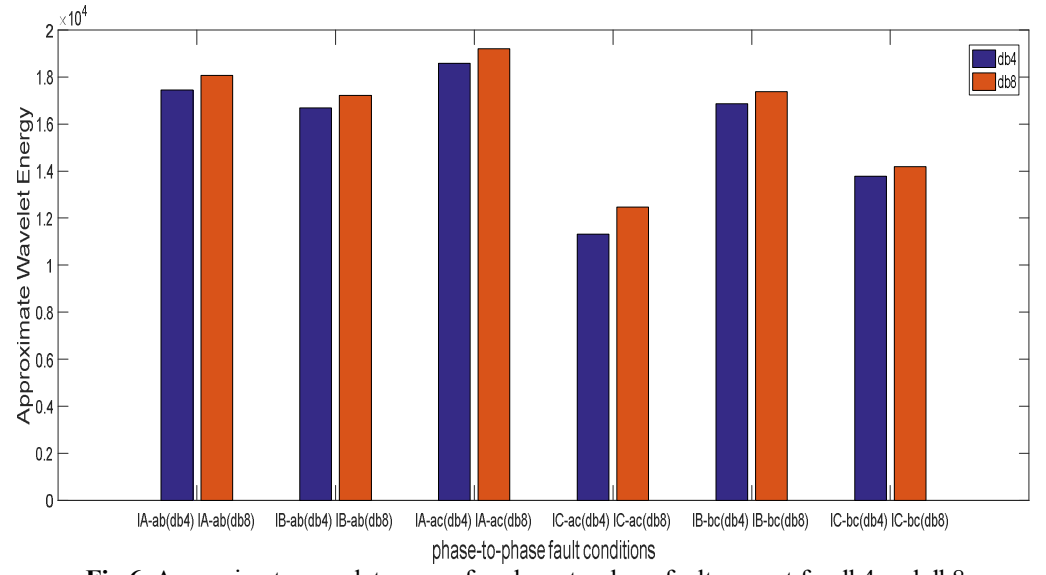

Fig 6. Approximate wavelet energy for phase-to-phase fault current for db4 and db8

Table 4: Normalized wavelet energy for inter-turn and phase-to-phase fault current signal

\begin{tabular}{ccccccccc}
\hline \multicolumn{8}{c}{ Table 4: Normalized wavelet energy for inter-turn and phase-to-phase fault current signal } \\
\hline$N_{E_{I a-i a}}$ & $N_{E_{I b-i b}}$ & $N_{E_{I c-i c}}$ & $N_{E_{I a-a b}}$ & $N_{E_{I b-a b}}$ & $N_{E_{I a-a c}}$ & $N_{E_{I c-a c}}$ & $N_{E_{I b-b c}}$ & $N_{E_{I c-b c}}$ \\
98.5172 & 98.5889 & 98.5981 & 98.6031 & 98.6658 & 98.6566 & 98.7017 & 98.6827 & 98.7191 \\
\hline
\end{tabular}

Table 6: Normalized WE obtained from the normal current

\begin{tabular}{ccc}
\multicolumn{3}{c}{ Healthy current normalised WE } \\
\hline$N_{I a-h}$ & $N_{I b-h}$ & $N_{I c-h}$ \\
20.95 & 36.64 & 36.04 \\
\hline
\end{tabular}

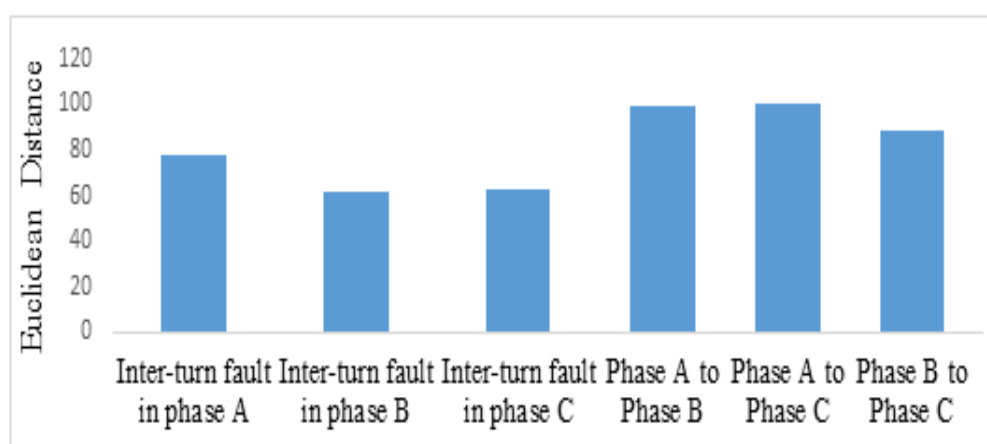

Fig 7: Euclidean Distance of different fault current type from reference value

It is also observed that the variation in the Euclidean Distance of the Wavelet Energy obtained for phase-tophase fault is higher than the inter-turn fault for the current signal. Based on the Euclidean distance

$$
K_{1}=0.60 \times 10^{2} K_{2}=0.80 \times 10^{2} \text { and } K_{3}=1.0 \times 10^{2}
$$$$
\therefore
$$

$$
\begin{aligned}
& \text { if } K_{2} \geq D_{f} \geq K_{1} \text { it can be identified as Inter-turn Fault } \\
& \text { if } K_{3} \geq D_{f}>K_{2} \text { it can be identified as Phase-to-Phase Fault }
\end{aligned}
$$

obtained from the wavelet energy of the fault and normal current signal, three classification points are set as; 
Conclusion: Stator fault classification has been presented in this paper. This technique distinguished between normal state, inter-turn fault and phase-tophase fault in induction motor using Discrete Wavelet Transform. Daubechies 8 mother wavelet proved to be efficient and was used in the classification. The results established that the technique for inter-turn and phaseto-phase faults detection and classification based on Euclidean Distance of the Wavelet Energy Coefficient of the stator current is effective and efficient. This will help in the diagnosis of induction motor stators fault.

\section{REFERENCES}

Abdullateef, AI; Fagbolagun, OS; Sanusi, MF (2019). Data Acquisition of Current and Vibration Data for Rewound Burnt Three-Phase Induction Motor. J. of Appl.Sci.\& Environ. Manage. 2 3(12): 21772183.

Bendjama, H; Boucherit, MS; Bouhouche, S (2013). Fault Diagnosis Of Rotating Machinery Using Wavelet Transform And Principal Component Analysis. IOSR J. of VLSI and Sig. Proce. 12-18.

Bhowmik, PS; Pradhan, S; Prakash, M (2013). Fault Diagnostic and Monitoring Methods of Induction Motor: A Review. Inter. J. of Appl. Cont., Elect. and Elect. Eng. 1(1): 1-18.

Bonaldi, EL; Ely, L; Oliveira, DLD; Guedes, J; Lambert-torresm, G; Eduardo, L (2012). Predictive Maintenance by Electrical Signature Analysis to Induction Motors.

Cusidó, J; Luis, J; Martínez, R (2011). Transient Analysis and Motor Fault Detection using the Wavelet Transform. intechopen (978-953-307185-5): 43-60.

Dubravko, MHZ (2015). Brief review of motor current signature analysis. 1(1): 15-26.

Glowacz, A; Glowacz, W; Glowacz, Z; Kozik, J (2018). Early fault diagnosis of bearing and stator faults of the single-phase induction motor using acoustic signals. Measurement, 113: 1-9.

Granda, D; Aguilar, WG; Arcos-aviles, D; Sotomayor, D (2017). Broken Bar Diagnosis for Squirrel Cage Induction Motors Using Frequency Analysis Based on MCSA and Continuous Wavelet Transform. mathematical and computational applications, 22(30): 1-15
Granda, D; Arcos-aviles, D; Sotomayor, D (2018). Analysis of signal processing techniques commonly used for broken bars detection on induction motors. Inter. J. of Comp. and Elect. Eng., 7(1): 1-6.

He, W; Zi, Y; Chen, B; Wu, F; He, Z (2015). Automatic fault feature extraction of mechanical anomaly on induction motor bearing using ensemble super-wavelet transform. mechanical systems and signal processing, 55: 257-480

Isermann, R (2015). Model-Based Fault Detection and Diagnosis - Status and Applications. (c).

Kessler, BM; Payne, GL; Polyzou, WN (2008). Wavelet Notes arXiv : nucl-th / 0305025v2 4 Dec 2003. 1-86.

Liang, H; Chen, Y; Liang, S; Wang, C (2018). applied sciences Fault Detection of Stator Inter-Turn Short-Circuit in PMSM on Stator Current and Vibration Signal. applied science article, 1677: 111

Liu, R; Yang, B; Zio, E; Chen, X (2018). Artificial intelligence for fault diagnosis of rotating machinery: A review. Mechanical Systems and Signal Processing, 108: 33-47.

Mallat, S (1989). A theory for multiresolution signal decomposition: the wavelet representation. 11(7): 674-693.

Mustafa, MO; Nikolakopoulos, G; Gustafsson, T; Case, AH; Winding, CS; Circuit, S (2012). A Fault Diagnosis Scheme for Three Phase Induction Motors based on Uncertainty Bounds. Inter. conf. on electrical machine, 1: 1596-1601.

Nagarajan, S; Reddy, SR (2013). Modeling and Simulation of Faulty Squirrel Cage Induction Motor Using Magnet. Inter. J. of Comp. and Elect. Eng., 2(5): 867-869

Palácios, RHC; da Silva, IN; Goedtel, A; Godoy, WF (2015). A comprehensive evaluation of intelligent classifiers for fault identification in three-phase induction motors. Electric Power Systems Research, 127: 249-258

Polikar, R (2008). The wavelet tutorial. Part IV. Multiresolution analysis: the discrete wavelet transform. 
Ray, PK; Panigrahi, BK; Rout, PK (2016). Detection of Faults in Power System Using Wavelet Transform and Independent Component Analysis. First Inter Conf. on Advan. Comp. Comm. \& Elect. Tech. (October): 1-5.

Sahraoui, M; Zouzou, S; Ghoggal, A; Guedidi, S (2010). A new method to detect inter-turn shortcircuit in induction motors. Paper presented at the The XIX Inter. Conf. on Elect. Machines.

Siddiqui, KM; Sahay, K; Giri, VK (2014). Health Monitoring and Fault Diagnosis in Induction Motor- A Review. Inter. J. of Adv. Res. in Elect., Elect. and Instrum. Energy, 3(1): 6549-6565.
Subha, M (2018). Artificial Intelligence Based Stator Winding Fault Estimation in Three Phase Induction Motor. 2018 Second Inter. Conf. on Elect., Comm. and Aero. Techn. (ICECA): 19291933.

Tangborn, A (2013). Wavelet Transforms in Time Series Analysis. 2(3): 123-142.

Verrucchi (2008). A review on fault diagnosis of induction machines. Latin American Applied Research, 38(2): 113-121. 